\title{
PSEUDO-ORBIT SHADOWING IN THE FAMILY OF TENT MAPS
}

\author{
ETHAN M. COVEN, ITTAI KAN AND JAMES A. YORKE
}

\begin{abstract}
We study the family of tent maps-continuous, unimodal, piecewise linear maps of the interval with slopes $\pm s, \sqrt{2} \leq s \leq 2$. We show that tent maps have the shadowing property (every pseudo-orbit can be approximated by an actual orbit) for almost all parameters $s$, although they fail to have the shadowing property for an uncountable, dense set of parameters. We also show that for any tent map, every pseudo-orbit can be approximated by an actual orbit of a tent map with a perhaps slightly larger slope.
\end{abstract}

1. Introduction. Let $f: X \rightarrow X$ be a continuous map of compact metric space to itself. The orbit of $x \in X$ is the sequence $\left\{x, f(x), f^{2}(x), \ldots\right\}$. Thus a sequence $\left\{x_{0}, x_{1}, \ldots\right\}$ is an orbit if and only if $f\left(x_{i}\right)=x_{i+1}$ for every $i \geq 0$. Given a number $\delta>0$, a $\delta$-pseudo-orbit is a sequence $\left\{x_{0}, x_{1}, \ldots\right\}$ such that $d\left(f\left(x_{i}\right), x_{i+1}\right) \leq \delta$ for every $i \geq 0$. The notion of pseudo-orbit goes back at least to G. D. Birkhoff [Bi]. Pseudo-orbits arise when noise is introduced into a dynamical system, for example, when orbits are calculated by a computer. In such cases, and especially for expanding maps, where errors propagate exponentially, it is important to know when pseudo-orbits can be approximated by actual orbits.

We say that $f$ has the shadowing property (also known as the pseudo-orbit tracing property) if for every $\varepsilon>0$, there is a $\delta>0$ such that every $\delta$-pseudo-orbit can be $\varepsilon$-shadowed by an actual orbit, i.e., if $\left\{x_{0}, x_{1}, \ldots\right\}$ satisfies $d\left(f\left(x_{i}\right), x_{i+1}\right) \leq \delta$ for every $i \geq 0$, then there is an $x \in X$ such that $d\left(f^{i}(x), x_{i}\right) \leq \varepsilon$ for all $i \geq 0$.

The term "shadowing" was introduced by R. Bowen, although the concept had appeared previously in the work of D. Anosov [A]. The fact that Axiom A diffeomorphisms (restricted to the nonwandering set) have the shadowing property is the key to the analysis of such maps [Bo]. For example, one gets structural stability from the shadowing property and expansiveness $[\mathbf{W}]$.

In this paper, we consider the family of tent maps, i.e., the piecewise linear maps $f_{s}:[0,2] \rightarrow[0,2], \sqrt{2} \leq s \leq 2$, defined by

$$
\begin{aligned}
f_{s}(x) & =s x, & & 0 \leq x \leq 1, \\
& =s(2-x), & & 1 \leq x \leq 2 .
\end{aligned}
$$

See the figure.

Restricting the parameters to lie in the interval $[\sqrt{2}, 2]$ is a technical convenience. Using the fact that for $1<s \leq \sqrt{2}, f_{s}^{2}$ (restricted to an appropriate interval) is

Received by the editors May 10, 1987.

1980 Mathematics Subject Classification (1985 Revision). Primary 58F30; Secondary 58F08, $58 \mathrm{~F} 15$ 


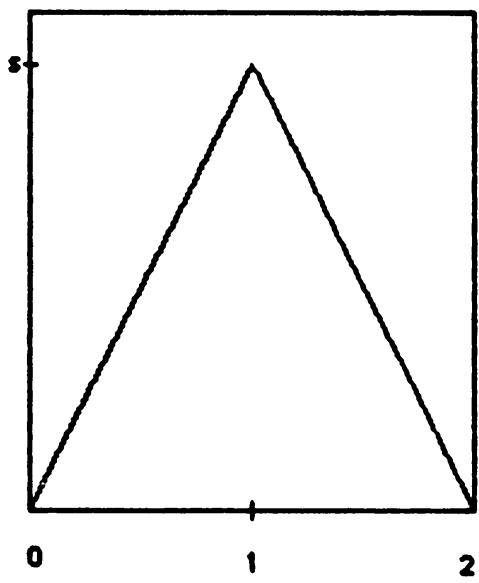

FIGURE

topologically conjugate to $f_{s^{2}}$, it can be shown that all the theorems (although not all the lemmas) of this paper hold for the parameter interval $(1,2]$.

The main results of this paper are a test for the shadowing property in tent maps (Theorem 4.2), theorems about the size of the set of parameters for which $f_{s}$ has the shadowing property (Theorems 6.1 and 7.4), and a theorem about shadowing pseudo-orbits of $f_{s}$ with actual orbits of $f_{t}$ (Theorem 8.1).

Recall that a point $x \in X$ is recurrent if for every $\varepsilon>0$, there is a positive integer $n$ such that $d\left(f^{n}(x), x\right) \leq \varepsilon$. Theorem 4.2 says roughly that for $s \neq 2, f_{s}$ has the shadowing property if and only if the critical point $c=1$ is recurrent and for every $\varepsilon>0$, there is positive integer $n$ such that $\left|f_{s}^{n}(c)-c\right| \leq \varepsilon$, and either $f_{s}^{n}(c)=c$ or $\left|f_{s}^{n}(x)-c\right|$ has a local maximum at $x=c$. The latter condition can be read off the kneading sequence of $f_{s}$. In particular, $f_{s}$ has the shadowing property if the critical point is periodic, e.g., if $s=(1+\sqrt{5}) / 2$, the Golden Mean, and does not if the critical point is preperiodic (i.e., has a periodic point in its orbit), but not periodic, e.g., if $s=\sqrt{2}$.

THEOREM 6.1. $f_{s}$ has the shadowing property for almost all parameters.

THEOREM 7.4. The set of parameters for which $f_{s}$ does not have the shadowing property is locally uncountable.

(A set is locally uncountable if its intersection with any open set is uncountable.)

If $f_{s}$ does not have the shadowing property, then there is an $\varepsilon>0$ such that for every $\delta>0$, there is a $\delta$-pseudo-orbit which cannot be $\varepsilon$-shadowed by any actual orbit. In fact, it can be shown that with probability one, a randomly chosen $\delta$ pseudo-orbit cannot be $\varepsilon$-shadowed by any actual orbit. By this we mean that, with $\varepsilon>0$ as above, for each $\delta>0$, there is a natural measure on the collection of all $\delta$-pseudo-orbits (cf., the uniform diffusion example in $[\mathbf{Y}]$ ) such that the set of $\delta$-pseudo-orbits which can be $\varepsilon$-shadowed by an actual orbit has measure zero.

Even if $f_{s}$ does not have the shadowing property, pseudo-orbits of $f_{s}$ can be shadowed by actual orbits of $f_{t}$ for some nearby $t \geq s$. 
THEOREM 8.1. Given a parameter $s$ and an $\varepsilon>0$, there is $a \delta>0$ and a parameter $t \geq s$ such that every $\delta$-pseudo-orbit of $f_{s}$ can be $\varepsilon$-shadowed by an actual orbit of $f_{t}$.

Pseudo-orbit shadowing in more general families of unimodal maps has been studied by H. Nusse and the third author [NY]. They obtain a variant of Theorem 8.1 using other methods.

2. Shadowing: generalities. In this section, $f$ and $g$ are continuous maps of a compact metric space $X$ to itself, and $B(x, \varepsilon)=\{y \in X: d(x, y) \leq \varepsilon\}$ is the closed $\varepsilon$-ball about $x \in X$, where $d$ is the metric on $X$.

Our positive results are based on variants of the following elementary observation.

LEMMA 2.1. Let $\varepsilon, \delta>0$. If $B(f(x), \varepsilon+\delta) \subseteq g[B(x, \varepsilon)]$ holds for all $x \in X$, then any $\delta$-pseudo-orbit of $f$ can be $\varepsilon$-shadowed by an actual orbit of $g$.

ProOF. Let $\left\{x_{0}, x_{1}, \ldots\right\}$ be a $\delta$-pseudo-orbit of $f$. Define sets $W_{0}, W_{1}, \ldots$ as follows:

$$
\left.W_{0}=B\left(x_{0}, \varepsilon\right), \quad W_{k}=W_{k-1} \cap g^{-k}\left[B\left(x_{k}\right), \varepsilon\right)\right], \quad k \geq 1 .
$$

By induction, $g^{k}\left(W_{k}\right)=B\left(x_{k}, \varepsilon\right)$ holds for all $k \geq 0$. Hence $\bigcap W_{k} \neq \varnothing$, and $\left\{x_{0}, x_{1}, \ldots\right\}$ can be $\varepsilon$-shadowed by the $g$-orbit of any point in $\bigcap W_{k}$.

It follows from Lemma 2.1 that

LEMMA 2.2. $f$ has the shadowing property if for every $\varepsilon>0$, there is a $\delta>0$ such that $B(f(x), \varepsilon+\delta) \subseteq f[B(x, \varepsilon)]$ holds for all $x \in X$.

It is easy to see that the condition of Lemma 2.2 is satisfied by the map $f(z)=z^{2}$ of the unit circle, as well as by the tent map $f_{2}$. However, the condition is not satisfied by any tent map except $f_{2}$. It fails at the critical point.

LEMMA 2.3. Let $\varepsilon, \delta>0, \lambda \geq 1$, and let $N$ be a positive integer. Suppose that for each $x \in X$, there is a positive integer $n=n(x) \leq N$ satisfying

$$
f\left[B\left(f^{n}(x), \varepsilon+\delta\right)\right] \subseteq\left\{f^{n+1}(y): d(x, y) \leq \varepsilon, d\left(f^{i}(x), f^{i}(y)\right) \leq \lambda \varepsilon, 1 \leq i \leq n\right\} .
$$

Then any $\delta^{\prime}$-pseudo-orbit can be $\varepsilon^{\prime}$-shadowed by an actual orbit, where $\varepsilon^{\prime} \geq \lambda \varepsilon+$ $\delta$ and $\delta^{\prime}$ is small enough so that for any $\delta^{\prime}$-pseudo-orbit $\left\{x_{0}, x_{1}, \ldots\right\}$, we have $d\left(f^{k}\left(x_{0}\right), x_{k}\right) \leq \delta$ for $1 \leq k \leq N$.

ProOF. For $x \in X$ and $n$ a positive integer, write

$$
A(x, n)=\left\{y: d(x, y) \leq \varepsilon, d\left(f^{i}(x), f^{i}(y)\right) \leq \lambda \varepsilon, 1 \leq i \leq n\right\} .
$$

Then we can rewrite $(2-1)$ as

$$
f\left[B\left(f^{n}(x), \varepsilon+\delta\right)\right] \subseteq f^{n+1}[A(x, n)] .
$$

Let $\left\{x_{0}, x_{1}, \ldots\right\}$ be a $\delta^{\prime}$-pseudo-orbit. Define integers $m_{k}$ and $n_{k}$, and sets $W_{k}$, $k \geq 0$, as follows.

$$
m_{0}=0, \quad n_{0}=n\left(x_{0}\right), \quad W_{0}=A\left(x_{m_{0}}, n_{0}\right),
$$


and for $k \geq 1$,

$$
\begin{aligned}
m_{k} & =m_{k-1}+n_{k-1}=n_{0}+\cdots+n_{k-1}, \\
n_{k} & =n\left(x_{m_{k}}\right) \\
W_{k} & =W_{k-1} \cap f^{-m_{k}-1}\left(f\left[A\left(x_{m_{k}}, n_{k}\right)\right]\right) .
\end{aligned}
$$

It suffices to show

$$
f^{m_{k}+1}\left(W_{k}\right)=f\left[A\left(x_{m_{k}}, n_{k}\right)\right], \quad k \geq 0 .
$$

For if $(2-3)$ is true, then $\left\{x_{0}, x_{1}, \ldots\right\}$ can be $\varepsilon^{\prime}$-shadowed by the orbit of any $y \in$ $\cap W_{k}$. To see this, fix $i \geq 0$.

If $i=0$, then $y \in W_{0} \subseteq B\left(x_{0}, \varepsilon\right)$. Thus $d\left(f^{0}(y), x_{0}\right) \leq \varepsilon^{\prime}$ since $\varepsilon \leq \varepsilon^{\prime}$. For $i \geq 1$, write $i=m_{k}+j$, where $k \geq 0$ and $0<j \leq n_{k}$. Then

$$
d\left(f^{i}(y), x_{i}\right) \leq d\left(f^{j+m_{k}}(y), f^{j}\left(x_{m_{k}}\right)\right)+d\left(f^{j}\left(x_{m_{k}}\right), x_{m_{k}+j}\right) .
$$

Now

$$
f^{j+m_{k}}(y)=f^{j-1}\left(f^{m_{k}+1}(y)\right) \in f^{j-1}\left[f^{m_{k}+1}\left(W_{k}\right)\right]=f^{j}\left[A\left(x_{m_{k}}, n_{k}\right)\right]
$$

and therefore $d\left(f^{j+m_{k}}(y), f^{j}\left(x_{m_{k}}\right)\right) \leq \lambda \varepsilon$. By our choice of $\delta^{\prime}$, we have

$$
d\left(f^{j}\left(x_{m_{k}}\right), x_{m_{k}+j}\right) \leq \delta .
$$

Hence, since $\varepsilon^{\prime} \geq \lambda \varepsilon+\delta$, we have $d\left(f^{i}(y), x_{i}\right) \leq \varepsilon^{\prime}$.

We verify (2-3) by induction on $k$. (2-3) holds for $k=0$ by definition. Suppose that $k \geq 0$ and (2-3) holds for $k$. Since

$$
f^{m_{k+1}+1}\left(W_{k+1}\right)=f^{m_{k+1}+1}\left(W_{k}\right) \cap f\left[A\left(x_{m_{k+1}}, n_{k+1}\right)\right],
$$

it suffices to show that $f\left[A\left(x_{m_{k+1}}, n_{k+1}\right)\right] \subseteq f^{m_{k+1}+1}\left(W_{k}\right)$. We have

$$
\begin{aligned}
f\left[A\left(x_{m_{k+1}}\right), n_{k+1}\right] & \subseteq f\left[B\left(x_{m_{k+1}}, \varepsilon\right)\right] \subseteq f\left[B\left(f^{n_{k}}\left(x_{m_{k}}\right), \varepsilon+\delta\right)\right] \subseteq f^{n_{k}+1}\left[A\left(x_{m_{k}}, n_{k}\right)\right] \\
& =f^{n_{k}}\left(f\left[A\left(x_{m_{k}}, n_{k}\right)\right]=f^{n_{k}}\left[f^{m_{k}+1}\left(W_{k}\right)\right]=f^{m_{k+1}+1}\left(W_{k}\right) .\right.
\end{aligned}
$$

The first containment is by our choice of $\delta^{\prime}$, and the second is by (2-2).

It follows from Lemma 2.3 that

LEMMA 2.4. $f$ has the shadowing property if there is a constant $\lambda \geq 1$ such that for every $\varepsilon>0$, there is a $\delta>0$ and a positive integer $N$ such that for each $x \in X$, there is a positive integer $n=n(x) \leq N$ satisfying

$$
f\left[B\left(f^{n}(x), \varepsilon+\delta\right)\right] \subseteq\left\{f^{n+1}(y): d(x, y) \leq \varepsilon, d\left(f^{i}(x), f^{i}(y)\right) \leq \lambda \varepsilon, 1 \leq i \leq n\right\} .
$$

To prove Theorem 8.1, about shadowing pseudo-orbits of $f_{s}$ with actual orbits of $f_{t}$, we will use the following lemma. Its proof, which we omit, is similar to those of Lemmas 2.1 and 2.3.

LEMMA 2.5. Let $\varepsilon, \delta>0$ and let $N$ be a positive integer. Suppose that for each $x \in X$, there is a positive integer $n=n(x) \leq N$ such that $B\left(f^{n}(x), \varepsilon+\delta\right) \subseteq$ $g^{n}[B(x, \varepsilon)]$. Then any $\delta^{\prime}$-pseudo-orbit of $f$ can be $\varepsilon^{\prime}$-shadowed by an actual orbit of $g$, where

$$
\begin{aligned}
\varepsilon^{\prime}=\delta & +\sup _{1 \leq k \leq N} \sup _{x \in X} \sup _{y \in B(x, \varepsilon)} d\left(g^{k}(x), g^{k}(y)\right) \\
& +\sup _{1 \leq k \leq N} \sup _{x \in X} d\left(f^{k}(x), g^{k}(x)\right)
\end{aligned}
$$


and $\delta^{\prime}$ is small enough so that for any $\delta^{\prime}$-pseudo-orbit $\left\{x_{0}, x_{1}, \ldots\right\}$ of $f$, we have $d\left(f^{k}\left(x_{0}\right), x_{k}\right) \leq \delta$ for $1 \leq k \leq N$.

3. Tent maps: generalities. We will see in the next section that some of the information needed to determine whether a tent map has the shadowing property can be expressed in terms of the kneading sequence [MT] of the map. In this section, we introduce enough terminology and notation to do just that.

Let $\nu=\nu(s)=\nu_{1} \nu_{2} \cdots$ be the kneading sequence of $f_{s}$, i.e., the (extended) itinerary of the critical value, defined as follows:

$$
\begin{array}{ll}
\nu_{n}=L & \text { if } f_{s}^{n}(c)<c \\
\nu_{n}=C & \text { if } f_{s}^{n}(c)=c \\
\nu_{n}=R & \text { if } f_{s}^{n}(c)>c
\end{array}
$$

Related to the kneading sequence is what we call the signature sequence $\sigma=$ $\sigma(s)=\sigma_{1} \sigma_{2} \ldots$, defined by

$$
\begin{gathered}
\sigma_{1}=-1, \\
\sigma_{n+1}=\sigma_{n} \quad \text { if } \nu_{n}=L, \\
\sigma_{n+1}=-1 \quad \text { if } \nu_{n}=C, \\
\sigma_{n+1}=-\sigma_{n}
\end{gathered}
$$

The reader familiar with kneading theory will recognize that $\sigma$ is the sequence of coefficients of the invariant coordinate of the critical value, repeated infinitely often if the critical point is periodic.

In the sequel, we shall encounter intervals where we know the endpoints, but not their order. We introduce the following notation: if $x \neq y$, then $\langle x, y\rangle$ denotes either $[x, y]$ or $[y, x]$, whichever make sense.

The following lemma is trivial, yet basic to the analysis which follows. Recall that $c=1$ is the common critical point of all the tent maps.

LEMMA 3.1. Given a parameter $s$, if $\varepsilon>0$ is sufficiently small, then:

(1) If $|x-c| \leq \varepsilon$, then $f_{s}[B(x, \varepsilon)]=B\left(f_{s}(x), s \varepsilon\right)$.

(2) If $z \in\langle x, y\rangle$ and $c \notin \operatorname{int}\langle x, y\rangle$, then $f_{s}(z) \in\left\langle f_{s}(x), f_{s}(y)\right\rangle$ and $\left|f_{s}(x)-f_{s}(y)\right|=$ $s|x-y|$.

(3) If $x \neq y$, then $c \in \operatorname{int}\left\langle f_{s}^{k}(x), f_{s}^{k}(y)\right\rangle$ for some $k \geq 0$.

It follows from Lemma 3.1 that given a parameter $s$, a positive integer $n$, and $\varepsilon>0$ sufficiently small, the endpoints of $f_{s}^{n}[c-\varepsilon, c+\varepsilon]$ are $f_{s}^{n}(c)$ and $f_{s}^{n}(c) \pm s^{n} \varepsilon$. The sign is given by the signature sequence.

LEMMA 3.2. Given a parameter $s$ and a positive integer $n$, if $|x-c|$ is small enough so that $c \notin \operatorname{int}\left[f_{s}^{k}\langle c, x\rangle\right]$ for $1 \leq k<n$, then $f_{s}^{n}(x)=f_{s}^{n}(c)+\sigma_{n}|x-c| s^{n}$. In particular, if $\varepsilon>0$ is sufficiently small, then $f_{s}^{n}[c-\varepsilon, c+\varepsilon]=\left\langle f_{s}^{n}(c), f_{s}^{n}(c)+\sigma_{n} s^{n} \varepsilon\right\rangle$.

4. A test for the shadowing property in tent maps. In this section, we obtain necessary and sufficient conditions for a tent map to have the shadowing property (Theorem 4.2). These conditions are that the critical point is recurrent and returns close to itself on the "correct side", a condition which can be stated in terms of the kneading and signature sequences. 
LEMMA 4.1. Let $s \neq 2$. Then $f_{s}$ has the shadowing property if and only if every $\varepsilon>0$, there is a positive integer $M$ such that $c \in\left\{f_{s}^{M}(y):\left|f_{s}^{i}(y)-f_{s}^{i}(c)\right| \leq \varepsilon\right.$, $0 \leq i \leq M\}$.

PROOF. Fix $s$ and write $f$ in place of $f_{s}$.

PART I. Suppose the condition holds, and let $\varepsilon>0$. We verify that the condition of Lemma 2.4 holds with $\lambda=s^{4}, \delta=(s-1) \varepsilon$, and $N=M+1$, i.e., for each $x \in[0,2]$, there is a positive integer $n=n(x) \leq M+1$ such that

$$
f\left[B\left(f^{n}(x), s \varepsilon\right)\right] \subseteq\left\{f^{n+1}(y):|x-y| \leq \varepsilon,\left|f^{i}(x)-f^{i}(y)\right| \leq s^{4} \varepsilon, 1 \leq i \leq n\right\}
$$

Note that (4-1) is implied by

$$
B\left(f^{n}(x), s \varepsilon\right) \subseteq\left\{f^{n}(y):|x-y| \leq \varepsilon,\left|f^{i}(x)-f^{i}(y)\right| \leq s^{4} \varepsilon, 1 \leq i \leq n\right\} .
$$

Suppose first that $f^{3}(c)=c$, i.e., $s=(1+\sqrt{5}) / 2$, the Golden Mean. Let $\varepsilon>0$ be small enough so that $c \notin f^{i}[c-\varepsilon, c+\varepsilon]$ for $i=1$ or 2 , and also $\left(s+s^{3}\right) \varepsilon<\frac{1}{2}$. It is easy to verify that (4-2) holds with

$$
\begin{array}{ll}
n(x)=1 & \text { if }|x-c| \geq \varepsilon, \\
n(x)=2 & \text { if } \varepsilon / s \leq|x-c|<\varepsilon, \\
n(x)=3 & \text { if } \varepsilon / s^{2} \leq|x-c|<\varepsilon / s .
\end{array}
$$

Now suppose that $|x-c| \leq \varepsilon / s^{2}$. Then $f^{3}(x) \in[c, c+s \varepsilon]$, and

$$
\left\{f^{3}(y):|x-y| \leq \varepsilon,\left|f^{i}(x)-f^{i}(y)\right| \leq s^{4} \varepsilon, 1 \leq i \leq 3\right\}=\left[c, f^{3}(x)+s^{3} \varepsilon\right] .
$$

But $f\left[B\left(f^{3}(x), s \varepsilon\right)\right]=f\left[c, f^{3}(x)+s \varepsilon\right]$, so (4-1) holds with $n(x)=3$. Similarly, the condition of Lemma 2.4 holds if $f^{4}(c)=c$.

We may therefore suppose that $f^{3}(c) \neq c$ and $f^{4}(c) \neq c$, and that $\varepsilon>0$ is small enough so that $c \notin f^{i}\left[c-s^{4} \varepsilon, c+s^{4} \varepsilon\right], 1 \leq i \leq 4$. For $n \geq 1$, let

$$
C_{n}=\left\{f^{n}(y):\left|f^{i}(y)-f^{i}(c)\right| \leq \varepsilon, 0 \leq i \leq n\right\} .
$$

We may also assume that $M$ is the least positive integer such that $c \in C_{M}$. Our choice of $\varepsilon$ insures that $M \geq 5$.

If $|x-c| \geq \varepsilon$, then (4-2) holds with $n(x)=1$. Now fix $x$ with $|x-c|<\varepsilon$. For $n \geq 1$, let

$$
\begin{gathered}
D_{n}=\left\{f^{n}(y):\left|f^{i}(x)-f^{i}(y)\right| \leq \varepsilon, 0 \leq i \leq n\right\} \\
E_{n}=\left\{f^{n}(y):|x-y| \leq \varepsilon,\left|f^{i}(x)-f^{i}(y)\right| \leq s^{4} \varepsilon, 1 \leq i \leq n\right\} .
\end{gathered}
$$

With this notation, (4-1) and (4-2) become

$$
\begin{gathered}
f\left[B\left(f^{n}(x), s \varepsilon\right)\right] \subseteq f\left(E_{n}\right) . \\
B\left(f^{n}(x), s \varepsilon\right) \subseteq E_{n} .
\end{gathered}
$$

Note that $D_{n}$ and $E_{n}$ are intervals and that

$$
\begin{aligned}
& \text { if }|x-c| s^{k} \leq \varepsilon \text { and } c \notin \operatorname{int} D_{1}, \ldots, \text { int } D_{k-1}, \text { then } \\
& D_{k}=\left\langle f^{k}(c), f^{k}(x)+\sigma_{k} \varepsilon\right\rangle \text { and } f^{k}(c)=f^{k}(x)-\sigma_{k}|x-c| s^{k} .
\end{aligned}
$$


Resuming the proof, suppose first that $c \notin D_{k}, 1 \leq k \leq M$. We claim that $|x-c| s^{M}>\varepsilon$. If not, then by (4-3), $D_{M}=\left\langle f^{M}(c), f^{\bar{M}}(c)+\sigma_{M}\left(|x-c| s^{M}+\varepsilon\right)\right\rangle$. Since $M$ is the least positive integer such that $c \in C_{M}$, we have, as in (4-3),

$$
C_{M}=\left\langle f^{M}(c), f^{M}(c)+\sigma_{M} \varepsilon\right\rangle
$$

and so $c \in C_{M} \subseteq D_{M}$, which is a contradiction. Therefore, $|x-c| s^{M}>\varepsilon$ if $c \notin D_{k}$, $1 \leq k \leq M$.

Let $k$ be the least positive integer such that $|x-c| s^{k}>\varepsilon$. Thus $1 \leq k \leq M$. From (4-3) and the fact that $c \notin D_{1}, \ldots, D_{k-1}$, we get that

$$
D_{k-1}=\left\langle f^{k-1}(x)-\sigma_{k-1}|x-c| s^{k-1}, f^{k-1}(x)+\sigma_{k-1} \varepsilon\right\rangle .
$$

Then, since $c \notin D_{k-1}, D_{k}=\left[f^{k}(x)-\varepsilon, f^{k}(x)+\varepsilon\right]$. Finally, since $c \notin D_{k}$,

$$
f\left(D_{k}\right)=\left[f^{k+1}(x)-s \varepsilon, f^{k+1}(x)+s \varepsilon\right] \subseteq E_{k+1},
$$

so $\left(4-2^{\prime}\right)$ holds with $n(x)=k+1$.

Now suppose that $5 \leq k \leq M$ is the least positive integer such that $c \in D_{k}$. Using (4-3) again, we have $\left\langle f^{k}(x), f^{k}(x)+\sigma_{k} \varepsilon\right\rangle \subseteq D_{k}$. Suppose for definiteness that $\sigma_{k}=+1$. We consider the cases, $f^{k}(x) \geq c$, and $f^{k}(x)<c$, separately.

Let $f^{k}(x) \geq c$. By the minimality of $k, c \notin D_{1}, \ldots, D_{k-1}$, and so $f^{k-1}(x)+$ $\sigma_{k-1} \varepsilon \in D_{k-1}$. Thus

$$
f^{k}(x)+s \varepsilon=f\left(f^{k-1}(x)+\sigma_{k-1} \varepsilon\right) \in f\left(D_{k-1}\right) \subseteq E_{k} .
$$

But $c \in E_{k}$, and so $\left[c, f^{k}(x)+s \varepsilon\right] \subseteq E_{k}$. Since $c \leq f^{k}(x) \leq c+\varepsilon$, we have $f\left[B\left(f^{k}(x), s \varepsilon\right]=f\left[c, f^{k}(x)+s \varepsilon\right]\right.$, and therefore $\left(4-1^{\prime}\right)$ holds with $n(x)=k$.

Finally, let $f^{k}(x)<c$. We have chosen $\varepsilon$ so small that $k \geq 5$. Since $c \in E_{k}$, we have $c \notin E_{k-4} \cup \cdots \cup E_{k-1}$. As above, $f^{k-4}(x)+\sigma_{k-4} \varepsilon \in D_{k-4}$,

$$
f^{k}(x)+s^{4} \varepsilon=f^{4}\left(f^{k-4}(x)+\sigma_{k-4} \varepsilon\right) \in f^{4}\left(D_{k-4}\right) \subseteq E_{k},
$$

and $\left[c, f^{k}(x)+s^{4} \varepsilon\right] \subseteq E_{k}$. But $s+2<s^{4}$ (since $s \geq \sqrt{2}$ ), and so

$$
f\left[B\left(f^{k}(x), s \varepsilon\right)\right] \subseteq f\left[c, f^{k}(x)+(s+2) \varepsilon\right] \subseteq E_{k} .
$$

Therefore (4-1') holds with $n(x)=k$.

PART II. Suppose the condition fails.

Suppose first that the critical point is not recurrent. There is an $\varepsilon>0$ such that

$$
\left|f^{n}(c)-c\right|>\varepsilon \text { for all } n \geq 1 \text {. }
$$

Then, given $0<\delta \leq \varepsilon$, let $\left\{x_{0}, x_{1}, \ldots\right\}$ be the $\delta$-pseudo-orbit defined as follows:

$$
\begin{aligned}
& x_{0}=c, \\
& x_{1}=f(c)+\delta, \\
& x_{k}=f^{k-1}\left(x_{1}\right), \quad k \geq 2 .
\end{aligned}
$$

Suppose that $\left\{x_{0}, x_{1}, \ldots\right\}$ can be $\varepsilon$-shadowed by the orbit of $y$. Let $n$ be the least positive integer such that $c \in\left\langle f^{n}(y), x_{n}\right\rangle$. (If no such integer exists, then by Lemma 3.1(3), $f(y)=x_{1}$, which is impossible since $x_{1}>s$, the maximum value of $f$.) Now $f(c) \in\left\langle f(y), x_{1}\right\rangle$ and $c \notin\left\langle f(y), x_{1}\right\rangle$. Therefore $f^{2}(c) \in\left\langle f^{2}(y), x_{2}\right\rangle$. But $x_{2}=f\left(x_{1}\right)$, so (assuming $n>2$ ), $c \in\left\langle f^{2}(y), x_{2}\right\rangle$. Continuing, we get $c, f^{n}(c) \in\left\langle f^{n}(y), x_{n}\right\rangle$, and therefore $\left|f^{n}(c)-c\right|<\varepsilon$, contradicting (4-4). 
Finally, suppose that the condition fails for $\varepsilon>0$ but that the critical point is recurrent. Recall from Part I of this proof that

$$
C_{n}=\left\{f^{n}(y):\left|f^{i}(y)-f^{i}(c)\right| \leq \varepsilon, 0 \leq i \leq n\right\} .
$$

Since $c \notin C_{n}$ for all $n \geq 1$, we have $C_{n}=\left\langle f^{n}(c), f^{n}(c)+\sigma_{n} \varepsilon\right\rangle$. Thus, if $\left|f^{n}(c)-c\right| \leq$ $\varepsilon$, we must have $\sigma_{n}=+1$ or -1 according as $\nu_{n}=R$ or $L$.

Given $0<\delta \leq \varepsilon$, we construct a $\delta$-pseudo-orbit $\left\{x_{0}, x_{1}, \ldots\right\}$ as follows. Since $c$ is recurrent, $\left|f^{k}(c)-c\right| \leq \delta$ for some $k \geq 1$. Let

$$
\begin{aligned}
x_{i} & =f^{i}(c), \quad 0 \leq i \leq k-1, \\
& =f^{i-k}(c), \quad i \geq k .
\end{aligned}
$$

Suppose that $\left\{x_{0}, x_{1}, \ldots\right\}$ can be $\varepsilon$-shadowed by the orbit of $y$. Then $\left\{x_{k}, x_{k+1}, \ldots\right\}$ can be $\varepsilon$-shadowed by the orbit of $f^{k}(y)$. Hence $f^{k+j}(y) \in C_{j}$ for all $j \geq 0$. In particular,

$$
\sigma_{j}=+1 \text { if } f^{k+j}(y)>f^{k}(c) \text { and } \sigma_{j}=-1 \text { if } f^{k+j}(y)<f^{k}(c) .
$$

By Lemma 3.1 again, $c \in\left\langle f^{j}(c), f^{k+j}(y)\right\rangle$ for some $j \geq 1$. Since $\left\langle f^{j}(c), f^{k+j}(y)\right\rangle \subseteq$ $C_{j}$, we have $\left|f^{j}(c)-c\right| \leq \varepsilon$. Therefore

$$
\sigma_{j}=+1 \quad \text { if } \nu_{j}=R, \quad \sigma_{j}=-1 \quad \text { if } \nu_{j}=L .
$$

Since $c \in\left\langle f^{j}(c), f^{k+j}(y)\right\rangle,(4-5)$ and (4-6) cannot both hold.

THEOREM 4.2. Let $s \neq 2$. Then $f_{s}$ has the shadowing property if and only if for every $\varepsilon>0$, there is a positive integer $n$ such that $\left|f_{s}^{n}(c)-c\right| \leq \varepsilon$, and either $\nu_{n}=C$, or $\sigma_{n}=+1$ or -1 , according as $\nu_{n}=L$ or $R$.

(In light of Lemma 3.2, the second condition may be thought of as saying that either $f_{s}^{n}(c)=c$, or $\left|f_{s}^{n}(x)-c\right|$ has a local maximum at $x=c$.)

PROOF. Write $f$ and $\sigma$ in place of $f_{s}$ and $\sigma(s)$. Let $\varepsilon>0$, and let $n$ be the least positive integer such that the condition in the statement of the theorem holds. If $f^{n}(c)=c$, then $f$ has the shadowing property by Lemma 4.1. Suppose then that $f^{n}(c) \neq c$. By Lemma 3.1,

$$
\left\{f^{n}(x):\left|f^{i}(x)-f^{i}(c)\right| \leq \varepsilon, 0 \leq i \leq n\right\}=\left\langle f^{n}(c), f^{n}(c)+\sigma_{n} \varepsilon\right\rangle .
$$

But the condition on $\sigma_{n}$ and the fact that $\left|f^{n}(c)-c\right| \leq \varepsilon$ imply that $c \in\left\langle f^{n}(c)\right.$, $\left.f^{n}(c)+\sigma_{n} \varepsilon\right\rangle$. By Lemma 4.1, $f$ has the shadowing property.

Conversely, suppose that $f$ has the shadowing property and let $\varepsilon>0$. Then, by Lemma 4.1, there is a least positive integer $n$ such that $c \in\left\{f^{n}(x):\left|f^{i}(x)-f^{i}(c)\right| \leq\right.$ $\varepsilon, 0 \leq i \leq n\}$. Then (4-7) holds and hence so does the condition on $\sigma_{n}$.

5. Varying the parameter. In order to show that $f_{s}$ has the shadowing property for almost all parameters, we will need to examine how the orbit of the critical point $c=1$ varies with $s$. To do this, we introduce the functions $\varphi_{n}$, $n \geq 1$, defined on $[\sqrt{2}, 2]$ by $\varphi_{n}(s)=f_{s}^{n}(c)$. Each $\varphi_{n}$ is continuous and piecewise polynomial.

We say that a parameter $s$ is periodic with period $n \geq 1$ if $f_{s}^{n}(c)=c$ (i.e., $\left.\varphi_{n}(s)=c\right)$, but $f_{s}^{k}(c) \neq c$ for $1 \leq k<n$. By Lemma 4.1, $f_{s}$ has the shadowing property whenever $s$ is periodic. The following two statements are immediate. 
LEMMA 5.1. The set of interior endpoints of the polynomial pieces of $\varphi_{n}$ is precisely the set of parameters with period less than $n$.

LEMMA 5.2. $\varphi_{n}$ is differentiable except at parameters with period less than $n$.

The main result of this section is

LEMMA 5.3. There is a positive constant $\lambda$ such that for every $s \in[\sqrt{2}, 2]$ and every $n>0$ such that $\varphi_{n}^{\prime}(s)$ exists,

$$
\lambda s^{n} \leq\left|\varphi_{n}^{\prime}(s)\right| \leq 3 s^{n} \quad \text { and } \quad \operatorname{sgn}\left(\varphi_{n}^{\prime}(s)\right)=-\sigma_{n} .
$$

ProOF. First notice that if $\varphi_{n+1}^{\prime}(s)$ exists, then

$$
\begin{aligned}
\varphi_{n+1}^{\prime}(s)=s \varphi_{n}^{\prime}(s)+\varphi_{n}(s) & \text { if } \nu_{n}(s)=L, \\
\varphi_{n+1}^{\prime}(s)=-s \varphi_{n}^{\prime}(s)+\left(2-\varphi_{n}(s)\right) & \text { if } \nu_{n}(s)=R .
\end{aligned}
$$

If $\nu_{n}(s)=L$, then $\left|\varphi_{n}(s)\right| \leq 1$, and if $\nu_{n}(s)=R$, then $\left|2-\varphi_{n}(s)\right| \leq 1$. Hence,

$$
\begin{aligned}
\left|\varphi_{n+1}^{\prime}(s)\right| & \leq s\left|\varphi_{n}^{\prime}(s)\right|+1 \leq s\left|\left(s\left|\varphi_{n-1}^{\prime}(s)\right|\right)+1\right|+1 \leq \cdots \\
& \leq s^{n} \varphi_{1}^{\prime}(s)+s^{n-1}+\cdots+1 \leq s^{n+1} /(s-1) .
\end{aligned}
$$

Therefore, since $1 /(s-1)<3$, the right-hand inequality of $(5-1)$ holds.

If for some $\beta>0$, we have

$$
\left|\varphi_{n}^{\prime}(s)\right| \geq \beta+1 /(s-1) \text { and } \operatorname{sgn}\left(\varphi_{n}^{\prime}(s)\right)=-\sigma_{n},
$$

then it follows that

$$
\left|\varphi_{n+1}^{\prime}(s)\right| \geq s \beta+1 /(s-1) \text { and } \operatorname{sgn}\left(\varphi_{n+1}^{\prime}(s)\right)=-\sigma_{n+1} .
$$

The patient reader may verify that there is a $\beta>0$ such that for all $s \in[\sqrt{2}, 2]$, (5-3) holds with $n=8$, that $\left\{\left|\varphi_{n}^{\prime}(s)\right|: s \in[\sqrt{2}, 2], 1 \leq n \leq 7\right\}$ is bounded away from zero, and that the equality in (5-2) holds for $1 \leq n \leq 8$. Therefore, the existence of $\lambda$ follows, and so (5-1) holds.

It follows from Lemma 5.3 and the Mean Value Theorem that

LEMMA 5.4. Let $\sqrt{2} \leq a<2$. Then any subinterval of $[a, 2]$ of length greater than $2 / \lambda a^{n}$ contains a periodic parameter with period less than $n$, where $\lambda$ is the constant in Lemma 5.3 .

LEMMA 5.5. The periodic parameters are dense.

(Lemma 5.5 is not new. It follows easily from the fact that the map $s \mapsto \nu(s)$ is one-to-one, and the continuity of the maps $\varphi_{n}$.) The Mean Value Theorem and the fact that $\varphi_{n}^{\prime}$ is never zero also yield

LEMMA 5.6. If $f_{s}^{n}(c)=f_{t}^{n}(c)=c$, then there is a periodic parameter between $s$ and $t$ with period less than $n$. In particular, in any parameter interval, there is exactly one periodic parameter with smallest period.

6. $f_{s}$ has the shadowing property for almost all parameters. In this section, we prove that the set of parameters $s$ for which $f_{s}$ has the shadowing property has full Lebesgue measure (denoted by $\mu$ ) and is residual. 
THEOREM 6.1. $f_{s}$ has the shadowing property for almost all parameters.

PROOF. For $\varepsilon>0$, let $T_{\varepsilon}$ be the set of parameters in $[\sqrt{2}, 2]$ satisfying the conditions of Theorem 4.2, i.e., $s \in T_{\varepsilon}$ if and only if there is a positive integer $n$ such that $\left|f_{s}^{n}(c)-c\right| \leq \varepsilon$, and either $\nu_{n}=C$, or $\sigma_{n}=+1$ or -1 according as $\nu_{n}=L$ or $R$. It suffices to show that $T_{\varepsilon}$ has full measure if $0<\varepsilon<3 / \lambda$, where $\lambda$ is the constant in Lemma 5.3. Fix such an $\varepsilon>0$ and write $T$ in place of $T_{\varepsilon}$.

To show that $T$ has full measure, we show that for every closed interval $J \subseteq$ $(\sqrt{2}, 2)$,

$$
\mu(J \cap T) / \mu(J) \geq \lambda \varepsilon / 18 .
$$

This is sufficient, for then the complement of $T$ can have no point of density (Lebesgue point). It suffices to prove (6-1) for intervals $J=[a, b]$ such that there is a positive integer $N=N(a, b) \geq 4$ satisfying

$$
2 \varepsilon / 3 b^{N}+2 / \lambda a^{N} \leq b-a \leq 3 / \lambda a^{N}
$$

and

$$
(a / b)^{N} \geq 1 / 2
$$

and to prove that for any such interval

$$
\mu(J \cap T) \geq \varepsilon / 3 b^{N} .
$$

Any interval contained in $(\sqrt{2}, 2)$ can be decomposed into subintervals (i.e., written as a possibly infinite union of subintervals with disjoint interiors) satisfying (6-2) and (6-3), with different $N$ 's for different subintervals, and if (6-2), (6-3), and (6-4) hold for $J=[a, b]$, then

$$
\mu(J \cap T) / \mu(J) \geq\left(\varepsilon / 3 b^{N}\right) /\left(3 / \lambda a^{N}\right)=(\lambda \varepsilon / 9)(a / b)^{N} \geq \lambda \varepsilon / 18 .
$$

Let $J=[a, b]$ be such an interval, and write $\gamma$ in place of $\varepsilon / 3 b^{N}$. By Lemmas 5.2 and 5.3, if $s \in J$ has period $k<N$, then $\left|f_{s}^{k}(c)-c\right| \leq \varepsilon$ for all $t \in J$ such that $|t-s| \leq \gamma$. Write $J=J_{1} \cup J_{2} \cup J_{3}$, where

$$
J_{1}=[a, a+\gamma), \quad J_{2}=[a+\gamma, b-\gamma], \quad J_{3}=(b-\gamma, b] .
$$

Let $s_{0}$ be the periodic parameter in $J_{2}$ with smallest period, say $M$. Since the length of $J_{2}$ is greater than $2 / \lambda a^{N}$, we have $M<N$ by Lemma 5.4. Suppose that there are no periodic parameters in $J_{1} \cup J_{3}$ with period less than $M$. Then on $\left[s_{0}-\gamma, s_{0}+\gamma\right], \nu_{M-1}$ and $\sigma_{M}$ are constant, and $\varphi_{M}^{\prime}$ does not change sign. Therefore

$$
\sigma_{M}=+1 \text { or }-1 \text { according as } \nu_{M-1}=L \text { or } R
$$

holds either for all $s \in\left[s_{0}-\gamma, s_{0}\right]$, in which case $\left[s_{0}-\gamma, s_{0}\right] \subseteq T$, or for all $s \in\left[s_{0}, s_{0}+\gamma\right]$, in which case $\left[s_{0}, s_{0}+\gamma\right] \subseteq T$. In either case, $\mu(J \cap T) \geq \gamma$.

Suppose that there are (necessarily finitely many) periodic parameters in $J_{1} \cup J_{3}$ with period less than $M$. We construct finite sequences $\left\{p_{1}, \ldots, p_{n}\right\},\left\{q_{1}, \ldots, q_{n}\right\}$, and $\left\{s_{1}, \ldots, s_{n}\right\}$, with $s_{i} \in J_{1} \cup J_{3}$ having period less than $M$, such that

(6-5) $a \leq p_{k} \leq p_{k+1} \leq q_{k+1} \leq q_{k} \leq b$.

(6-6) $\left(a, p_{k}\right) \cup\left(q_{k}, b\right) \subseteq T$.

(6-7) If either $\left[s_{n}-\gamma, s_{n}\right] \subseteq\left(p_{n}, q_{n}\right) \cap T$ or $\left[s_{n}, s_{n}+\gamma\right] \subseteq\left(p_{n}, q_{n}\right) \cap T$, then the sequences have length $n$. 
(6-8) If the sequences have length $n$ and (6-7) does not hold for $n$, then there are no periodic parameters in $\left(p_{n}, q_{n}\right) \cap\left(J_{1} \cup J_{3}\right)$ with period less than $M$.

Assuming such sequences exist, if (6-7) holds for some $n$, then there is nothing to prove. If not, and the sequences have length $n$, then by (6-8), $s_{0}$ is the periodic parameter in $\left(p_{n}, q_{n}\right)$ with smallest period. Then either $\left[s_{0}-\gamma, s_{0}\right] \cap\left[p_{n}, s_{0}\right] \subseteq T$ or $\left[s_{0}, s_{0}+\gamma\right] \cap\left[s_{0}, q_{n}\right] \subseteq T$. Without loss of generality, assume the former. If $s_{0}-\gamma \geq p_{n}$, then $\mu(J \cap T) \geq \gamma$, while if $s_{0}-\gamma<p_{n}$, then $\left(a, s_{0}\right] \subseteq T$, and so $\mu(J \cap T) \geq s_{0}-a \geq \gamma$.

To construct these sequences, let $p_{1}=a$ and $q_{1}=b$. Assuming (6-7) does not hold for $n=1$, let $s_{1}$ be the periodic parameter in $\left(p_{1}, q_{1}\right)$ with smallest period. We must have either $s_{1} \in J_{1}$ and $s_{1}-\gamma<p_{1}$, or $s_{1} \in J_{3}$ and $s_{1}+\gamma>q_{1}$. In the first case, let $p_{2}=s_{1}$ and $q_{2}=q_{1}$, and in the second case, let $p_{2}=p_{1}$ and $q_{2}=s_{1}$. Notice that (6-6) holds for $k=2$. Repeat the procedure on $\left(p_{2}, q_{2}\right)$, etc.

COROLLARY 6.2. The set of parameters for which $f_{s}$ has the shadowing property is residual.

PROOF. In the proof of Theorem 6.1, we showed that given small enough $\varepsilon>0$ and a closed interval $J \subseteq(\sqrt{2}, 2)$ there is an open interval $J^{\prime} \subseteq J \cap T_{\varepsilon}$. Therefore, each $T_{\varepsilon}$ contains a dense open set.

7. $f_{s}$ does not have the shadowing property for a locally uncountable set of parameters. In this section, we show that the set of parameters $s \in[\sqrt{2}, 2]$ for which $f_{s}$ does not have the shadowing property is locally uncountable, i.e., it meets every open interval in an uncountable set. By Theorem 4.2, it is sufficient to exhibit, for each closed interval $J \subseteq(\sqrt{2}, 2)$, an uncountable set of parameters $s \in J$ such that for each such $s$, the critical point $c=1$ is not $f_{s}$-recurrent. To do this, we go back to the kneading theory.

The shift map $\tau$ from the space of all sequences over some finite alphabet to itself is defined by

$$
[\tau(\nu)]_{i}=\nu_{i+1}, \quad i \geq 1 .
$$

LEMMA 7.1. The critical point is $f_{s}$-recurrent if and only if $\nu(s)$ is $\tau$-recurrent.

The proof is an easy exercise, using the following combinatorial test for $\tau$ recurrence: $\nu$ is $\tau$-recurrent if and only if every initial word of $\nu$ appears at least twice in $\nu$.

In the kneading theory, sequences are ordered by the following rules.

(7-1) $L<C<R$.

(7-2) If $\nu=\nu_{1} \nu_{2} \ldots, \mu=\mu_{1} \mu_{2} \ldots$, and $n$ is the least positive integer such that $\nu_{n} \neq \mu_{n}$, then $\nu<\mu$ if either $\nu_{n}<\mu_{n}$ and there are an even number of $R$ 's in $\nu_{1} \cdots \nu_{n-1}$, or $\nu_{n}>\mu_{n}$ and there are an odd number of $R$ 's in $\nu_{1} \cdots \nu_{n-1}$.

With this order on sequences, the map $s \mapsto \nu(s)$ is increasing, and thus the kneading sequence of $f_{s}$ satisfies $\nu(s) \geq \nu(\sqrt{2})$, i.e., $\nu(s) \geq R L R^{\infty}$.

A sequence $\nu=\nu_{1} \nu_{2} \ldots$ is strictly maximal if $\tau^{n}(\nu)<\nu$ for all $n \geq 1$; it is primary (i.e., not a $*$-product) if there is no $k>1$ such that, except for $i \equiv 0$ $\bmod k, \nu_{i}=\nu_{j}$ whenever $i \equiv j \bmod k$. Note that a periodic parameter cannot have a strictly maximal kneading sequence. 
LEMMA 7.2 [DGP, Theorem 2; CE, p. 147 and Lemma III.1.6]. If $\nu>R L R^{\infty}$ is strictly maximal and primary, then $\nu$ is the kneading sequence of $f_{s}$ for some $s \in[\sqrt{2}, 2]$.

We call a parameter $s$ prefixed if the nonzero fixed point of $f_{s}$ is in the $f_{s}$-orbit of the critical point. Prefixed parameters are characterized by having kneading sequences of the form $W R^{\infty}$, where $W$ is a finite word with letters $R$ and $L$.

LEMMA 7.3. The prefixed parameters are dense in $[\sqrt{2}, 2]$.

ProOF. Let $J \subseteq(\sqrt{2}, 2)$ be an open interval. Choose $s \in J$ so that the critical point is not $f_{s}$-periodic, and let $z$ be the nonzero fixed point of $f_{s}$.

There is a positive integer $N$ such that if $t \in(\sqrt{2}, 2)$ and $\nu(t)$ agrees with $\nu(s)$ in the first $N$ places, then $t \in J$. There is an $\varepsilon>0$ such that if $|x-c| \leq \varepsilon$, then the itinerary of $f_{s}(x)$ agrees with $\nu(s)$ in the first $N$ places.

Now $\bigcup_{n>0} f_{s}^{-n}(z)$ is dense in $\left[f_{s}^{2}(c), f_{s}(c)\right]$, so there exists $x \in[c-\varepsilon, c+\varepsilon]$ and $n>0$ such that $f_{s}^{n}(x)=z$. We may choose $x$ so that

(7-3) $f_{s}(x) \neq z$.

(7-4) $\left|f_{s}^{k}(x)-c\right|>|x-c|$ for $k=1,2, \ldots$

Let $\xi$ be the $f_{s}$-itinerary of $f_{s}(x)$. We may also choose $x$ so that

$(7-5) \xi>R L R^{\infty}$.

$(7-6) \xi$ is primary.

(7-5) may be satisfied by choosing $x$ close enough to the critical point, and (7-6) may be satisfied by choosing $x$ so that $\operatorname{GCD}\left\{|j-k|: \xi_{j}=\xi_{k}=L\right\}=1$.

Then $\xi$ has the form $W R^{\infty}$. By (7-3) and (7-4), it is strictly maximal. Then by (7-5) and (7-6), it satisfies the hypotheses of Lemma 7.2. Therefore there exists $t \in(\sqrt{2}, 2)$ such that $\xi=\nu(t)$. Clearly $t$ is prefixed, and our choice of $\varepsilon$ and $N$ implies that $t \in J$.

THEOREM 7.4. The set of parameters for which $f_{s}$ does not have the shadowing property is locally uncountable.

ProOF. Let $J \subseteq[\sqrt{2}, 2]$ be an open interval. By Lemma $7.3, J$ contains a prefixed parameter $s$. The kneading sequence $\nu(s)$ may be written $W R^{\infty}$, where $W$ is a finite word with letters $R$ and $L$. We may choose $W$ to be long enough so that it contains an odd number of $R$ 's and so that if $\nu(t)$ begins with $W$, then $t \in J$.

We construct uncountably many sequences $\nu>R L R^{\infty}$ which are strictly maximal, primary, begin with $W$, and are not $\tau$-recurrent. Then by Lemma 7.2, any such $\nu$ is $\nu(t)$ for some $t \in J$; by Lemma 7.1, the critical point is not $f_{t}$-recurrent; and by Theorem $4.2, f_{t}$ does not have the shadowing property.

Let $n$ be the length of $W$. Since $\nu(s)=\nu_{1} \nu_{2} \cdots$ is strictly maximal, for each $k \geq 1$, there is a least positive integer $m=m(k)$ such that $\nu_{k} \neq \nu_{k+m}$. Since $\nu$ begins with $R L, m(k)=2$ for $k \geq n$. Let

$$
m=\max \{m(k): k \geq 1\}=\max \{m(k): 1 \leq k \leq n-1\} .
$$

Choose $n_{1}<n_{2}<\cdots$ with each $n_{i}$ odd and $n_{1}>m$. Then $\nu=W R^{n_{1}} W R^{n_{2}} \ldots$ is strictly maximal, not $\tau$-recurrent, and satisfies $\nu \geq R L R^{\infty}$. With the proper choice of finitely many $n_{i}$ 's, $\nu$ will be primary. There are uncountably many such $\nu$. 
With a bit more care, it can be shown that each interval contains uncountably many parameters such that the critical point is $f_{s}$-recurrent, yet $f_{s}$ does not have the shadowing property. With notation as in the proof above, define $\nu=" \lim " B_{k}$, where

$$
B_{1}=W R^{n_{1}}, \quad B_{k}=B_{k-1} B_{k-1} W R^{n_{k}}, \quad k \geq 2 .
$$

Then $\nu$ is strictly maximal, $\tau$-recurrent, primary, and satisfies $\nu>R L R^{\infty}$. Thus $\nu=\nu(t)$ for some $t \in J$ for which the critical point is $f_{t}$-recurrent. However, it can be shown that for $\varepsilon>0$ small enough, $\left|f_{t}^{n}(c)-c\right| \leq \varepsilon$ only when $n$ corresponds to the last entry in an appearance of some $B_{k}$. For any such $n, \nu_{n}=R$ and $\sigma_{n}=+1$. By Theorem 4.2, $f_{t}$ does not have the shadowing property.

8. Shadowing with parameter-shifting. Even if $f_{s}$ does not have the shadowing property, pseudo-orbits of $f_{s}$ can be shadowed by actual orbits of $f_{t}$ for some nearby $t \geq s$. In particular, we have

THEOREM 8.1. Given a parameter $s$ and an $\varepsilon>0$, there is $a \delta>0$ and a parameter $t \geq s$ such that every $\delta$-pseudo-orbit of $f_{s}$ can be $\varepsilon$-shadowed by an actual orbit of $f_{t}$.

PROOF. Fix $s<2$. (If $s=2$, choose $t=s$, for $f_{2}$ has the shadowing property by Lemma 2.2.) By Lemma 5.3, there is a positive constant $\lambda$ such that for all $r \in[\sqrt{2}, 2]$, and all $n>0$ such that $\varphi_{n}^{\prime}(r)$ exists

$$
\lambda r^{n} \leq\left|\varphi_{n}^{\prime}(r)\right| \leq 3 r^{n} \text { and } \operatorname{sgn}\left(\varphi_{n}^{\prime}(r)\right)=-\sigma_{n}(r) .
$$

Without loss of generality, $\lambda<1$. Choose $N$ is so large that

$$
S^{N}>5 / \lambda \text {. }
$$

If $s$ is a periodic parameter, then by Theorem $4.2, f_{s}$ has the shadowing property, and we choose $t=s$. Otherwise, let $\varepsilon>0$ be small enough so that $s+\varepsilon / 4<2$, and

$$
\left|f_{s}^{k}(c)-c\right|>2^{N+2} \varepsilon \text { for } 1 \leq k<N,
$$

and there is no periodic parameter in $[s, s+\varepsilon / 4]$ with period less than $N$. Choose $t=s+\varepsilon / 4$ and $\delta=\varepsilon / 4$.

It suffices to find $\varepsilon^{\prime}, \delta^{\prime}>0$ with $\varepsilon^{\prime} \rightarrow 0$ as $\varepsilon \rightarrow 0$ such that every $\delta^{\prime}$-pseudo-orbit of $f_{s}$ can be $\varepsilon^{\prime}$-shadowed by an actual orbit of $f_{t}$. We do this by verifying the hypothesis of Lemma 2.5 holds with $f=f_{s}, g=f_{t}$, and $\varepsilon, \delta$, and $N$ as above. That is, for each $x \in[0,2]$, we find a positive integer $n=n(x) \geq N$ such that

$$
B\left(f_{s}^{n}(x), \varepsilon+\delta\right) \subseteq f_{t}^{n}[B(x, \varepsilon)] .
$$

If $|x-c| \geq \varepsilon$, then (8-4) holds with $n(x)=1$. We fix $x$ with $|x-c|<\varepsilon$, and show that (8-4) holds with $n(x)=N$. By the symmetry of the tent maps about the critical point, we may assume that $x \geq c$.

By Lemma $5.2, \varphi_{k}$ is differentiable on $[s, t]$ for $1 \leq k \leq N$. Then by the Mean Value Theorem and (8-1),

$$
\left|f_{t}^{k}(c)-f_{s}^{k}(c)\right| \leq 3(t-s) t^{k}<2^{N-1} \varepsilon .
$$

Then, by (8-3), we have $c \notin\left\langle f_{s}^{k}(c), f_{t}^{k}(c)\right\rangle$ for $1 \leq k<N$. Also $\varphi_{N}^{\prime}$ exists and is never zero on $[s, t]$, so we have

$$
\sigma_{N} \text { is constant on }[s, t] .
$$


Let $y \in B(x, \varepsilon)$ and $1 \leq k<N$. Then $|x-y| \leq \varepsilon$ and $|y-c| \leq 2 \varepsilon$. It follows from Lemma 3.2 that

$$
\left|f_{t}^{k}(x)-f_{t}^{k}(y)\right| \leq t^{k} \varepsilon<2^{N-1} \varepsilon \quad \text { and } \quad\left|f_{t}^{k}(y)-f_{t}^{k}(c)\right| \leq 2 t^{k} \varepsilon<2^{N+1} \varepsilon .
$$

By (8-3), (8-5), and (8-7), we have

$$
c \notin f_{t}^{k}[B(x, \varepsilon)] \quad \text { for } 1 \leq k<N \text {. }
$$

Without loss of generality, we may assume that $\sigma_{N}=+1$, and hence that $\varphi_{N}^{\prime}<0$ on $[s, t]$. Using (8-6) and (8-8), and the fact that $f_{t}^{N}[B(x, \varepsilon)]=f_{t}^{N}[c, x+\varepsilon]$, we have, by Lemma 3.2 , that

$$
f_{t}^{N}[B(x, \varepsilon)]=\left[f_{t}^{N}(c), f_{t}^{N}(c)+(x+\varepsilon-c) t^{N}\right] .
$$

Similarly,

$$
B\left(f_{s}^{N}(x), \varepsilon+\delta\right)=\left[f_{s}^{N}(c)+(x-c) s^{N}-5 \varepsilon / 4, f_{s}^{N}(c)+(x-c) s^{N}+5 \varepsilon / 4\right] .
$$

By the Mean Value Theorem, $f_{t}^{N}(c)=f_{s}^{N}(c)+(t-s) \varphi_{N}^{\prime}(r)$ for some $r \in[s, t]$. But $\varphi_{N}^{\prime}<0$ on $[s, t]$. Therefore, using (8-1) and (8-2), we get

$$
\begin{aligned}
& f_{t}^{N}(c) \geq f_{s}^{N}(c)-3(t-s) t^{N} \geq f_{s}^{N}(c)-3 t^{N} \varepsilon / 4 \text { and } \\
& f_{t}^{N}(c) \leq f_{s}^{N}(c)-(t-s) \lambda s^{N}<f_{s}^{N}(c)-5 \varepsilon / 4 .
\end{aligned}
$$

Since $x \geq c$, it follows that

$$
f_{t}^{N}(c)<f_{s}^{N}(c)-5 \varepsilon / 4<f_{s}^{N}(c)+(x-c) s^{N}-5 \varepsilon / 4,
$$

i.e., the left-hand endpoint of $f_{t}^{N}[B(x, \varepsilon)]$ is less than the left-hand endpoint of $B\left(f_{s}^{N}(x), \varepsilon+\delta\right)$. It remains to check that the right-hand endpoints have the opposite order, i.e., that

$$
f_{t}^{N}(c)+(x+\varepsilon-c) t^{N}>f_{s}^{N}(c)+(x-c) s^{N}+5 \varepsilon / 4 .
$$

We calculate as follows:

$$
\begin{aligned}
f_{t}^{N}(c)+(x+\varepsilon-c) t^{N} & =f_{t}^{N}(c)+(x-c) t^{N}+t^{N} \varepsilon \\
& \geq f_{s}^{N}(c)+(x-c) s^{N}+t^{N} \varepsilon / 4 \quad \text { (by (8-9)) } \\
& >f_{s}^{N}(c)+(x-c) s^{N}+5 \varepsilon / 4,
\end{aligned}
$$

the final inequality by (8-2), since $\lambda<1$. This completes the proof.

ACKNOWLEDGMENTS. The authors thank H. Nusse and W. Reddy for useful conversations, and an anonymous friend for considerably shortening the proof of Lemma 7.3. This work was done while the first two authors were at the Institute for Advanced Study. They thank the Institute for its hospitality and support. The third author was partially supported by National Science Foundation Grant DMS 84-19910 and Air Force Office of Scientific Research Grant 81-0217.

\section{REFERENCES}

[A] D. V. Anosov, Geodesic flows on closed Riemann manifolds with negative curvature, Proc. Steklov Inst. Math. 90 (1967).

[Bi] G. D. Birkhoff, An extension of Poincaré's last geometric theorem, Acta Math. 47 (1925), 297-311. 
[Bo] R. Bowen, On Axiom A diffeomorphisms, CBMS Regional Conf. Ser. in Math., no. 35, Amer. Math. Soc., Providence, R.I., 1978.

[CE] P. Collet and J.-P. Eckmann, Iterated maps on the interval as dynamical systems, Progress in Physics 1, Birkhäuser, Boston, Mass., 1980.

[DGP] B. Derrid, A. Gervois, and Y. Pomeau, Iterations of endomorphisms on the real axis and representation of numbers, Ann. Inst. Henri Poincaré 29 (1978), 305-356.

[MT] J. Milnor and W. Thurston, On iterated maps of the interval, mimeographed notes, 1977.

[NY] J. Yorke and H. Nusse, Is every trajectory of some process near an exact trajectory of a nearby process?, preprint, 1986.

[W] P. Walters, On the pseudo-orbit tracing property and its relationship to stability, Lecture Notes in Math., vol. 668, Springer-Verlag, Berlin and New York, 1978, pp. 191-210.

[Y] L.-S. Young, Stochastic stability of hyperbolic attractors, preprint, 1985.

School of Mathematics, Institute for Advanced Study, Princeton, New JERSEY 08540

Department of Mathematics, Wesleyan University, Middletown, ConnectiCUT 06457 (Current address of E. M. Coven)

Institute for Physical SCIENCES AND TeChNology, UNiversity of MARYland, COllege PARK, MARYLAND 20742 (Current address of Ittai Kan and J. A. Yorke) 